\title{
Surface plasmon quantum cascade lasers as terahertz local oscillators
}

\author{
M. Hajenius, ${ }^{1,2}$ P. Khosropanah, ${ }^{2}$ J. N. Hovenier, ${ }^{1}$ J. R. Gao, ${ }^{1,2, *}$ T. M. Klapwijk, ${ }^{1}$ S. Barbieri, ${ }^{3}$ \\ S. Dhillon, ${ }^{3}$ P. Filloux, ${ }^{3}$ C. Sirtori, ${ }^{3}$ D. A. Ritchie, ${ }^{4}$ and H. E. Beere ${ }^{4}$ \\ ${ }^{1}$ Kavli Institute of NanoScience, Faculty of Applied Sciences, Delft University of Technology, Lorentzweg 1, 2628 CJ, \\ Delft, The Netherlands \\ ${ }^{2}$ SRON Netherlands Institute for Space Research, Groningen/Utrecht, The Netherlands \\ ${ }^{3}$ Matériaux et Phénomènes Quantiques, Université de Paris 7, 75205 Paris Cedex 13, France \\ ${ }^{4}$ Cavendish Laboratory, University of Cambridge, J.J. Thomas Avenue, Cambridge, CB3 oHE, United Kingdom \\ *Corresponding author: j.r.gao@tnw.tudelft.nl
}

Received November 13, 2007; revised December 16, 2007; accepted December 17, 2007; posted January 10, 2008 (Doc. ID 89666); published February 6, 2008

\begin{abstract}
We characterize a heterodyne receiver based on a surface-plasmon waveguide quantum cascade laser (QCL) emitting at $2.84 \mathrm{THz}$ as a local oscillator, and an $\mathrm{NbN}$ hot electron bolometer as a mixer. We find that the envelope of the far-field pattern of the QCL is diffraction-limited and superimposed onto interference fringes, which are similar to those found in narrow double-metal waveguide QCLs. Compared to the latter, a more directional beam allows for better coupling of the radiation power to the mixer. We obtain a receiver noise temperature of $1050 \mathrm{~K}$ when the mixer is at $2 \mathrm{~K}$, which, to our knowledge, is the highest sensitivity reported at frequencies beyond $2.5 \mathrm{THz}$. (C) 2008 Optical Society of America

OCIS codes: $140.3070,140.5960,040.0040$.
\end{abstract}

$\mathrm{THz}$ quantum cascade lasers (QCLs) [1] have been successfully demonstrated as local oscillators (LOs) in laboratory tests $[2,3]$ using either a double-metal (DM) or surface plasmon (SP) waveguide (W) QCL $[1,4]$. The far field of double-metal waveguide (DMW) QCLs is strongly divergent and dominated by a ringlike interference pattern [5]. The latter has been modeled [6] and is the consequence of: (i) the subwavelength lateral dimensions of the QCL cavity, and (ii) the interference due to the coherent emission from the facets and sides of the laser. Such a beam shape and phase front prevented an efficient coupling of the radiation to the mixer [2].

Surface plasmon waveguide (SPW) QCLs [1], where the active region is embedded between a heavily doped bottom layer and a top metal contact, can emit in a single mode despite the fact that the width of the cavity is typically larger than the wavelength of the radiation [7]. In this case a diffractionlimited single-spot beam is expected. Experimentally, low divergence single-spotlike beams have been observed in single mode [3] and multimode SPW QCLs [8].

In this Letter we study the beam patterns of two $2.8 \mathrm{THz}$ SPW QCLs with different widths. Contrary to the previous results, we find that the beam does not present a single maximum. Instead, we observe a ringlike interference pattern, superimposed onto a slow-varying envelope that follows the diffraction limit. Besides, we use one of the QCLs to perform a heterodyne experiment.

The QCLs used in this work are described in [9] and are based on a bound-to-continuum active region design and a SPW. The active region consists of $90 \mathrm{GaAs} / \mathrm{Al}_{0.15} \mathrm{Ga}_{0.85} \mathrm{As}$ repeated modules, giving a total thickness of $11.64 \mu \mathrm{m}$. The active layer is grown on the top of a $230 \mu \mathrm{m}$ thick semi-insulating GaAs substrate, sandwiched between a metallic top contact and a heavily $n$-doped GaAs bottom-contact channel. Therefore, the optical mode is not fully confined within the active region, but penetrates into the substrate down to a depth of $\sim 100 \mu \mathrm{m}$ at $2.8 \mathrm{THz}$ (see below). We used two laser ridges, which are $1500 \mu \mathrm{m}$ long, but $217 \mu \mathrm{m}$ and $158 \mu \mathrm{m}$ wide. The $217 \mu \mathrm{m}$ wide QCL is schematically shown in Fig. 1(a).

Since both QCLs showed similar far-field patterns, in this work we will focus on the $217 \mu \mathrm{m}$ wide device. Lasing spectra, measured at different bias currents using a Fourier-transform spectrometer with a resolution of $\sim 1 \mathrm{GHz}$, are displayed in Fig. 1(b), showing single mode emission at $2.835 \mathrm{THz}$. According to (a)

(c)
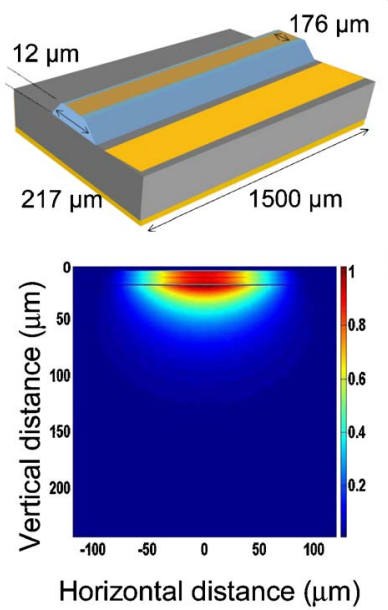

(b)

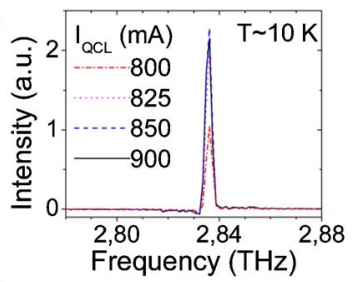

(d)

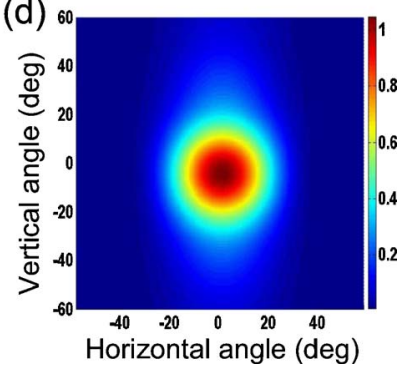

Fig. 1. (Color online) (a) Schematic view of the $217 \mu \mathrm{m}$ wide SP QCL used for the heterodyne experiment. The golden areas represent the metallic top and lateral contacts. (b) Emission spectra of the QCL. (c) Computed mode intensity at the front facet. (d) Computed far field beam pattern. 
higher resolution ( $\leqslant 1 \mathrm{MHz}$ ) spectra obtained on similar QCLs using a heterodyne technique in [10], we expect no higher-order lateral modes in this laser. The measured maximum output power in continuous wave (cw) mode was about $1.5 \mathrm{~mW}$ when it was operated at $6 \mathrm{~V}$ and $900 \mathrm{~mA}$, and at an operating temperature of $\sim 20 \mathrm{~K}$.

We measured the far-field beam pattern using the same setup described in [5]. The radiation intensity was measured using a pyrodetector with an aperture of $2 \mathrm{~mm}$ in diameter, placed at a radial distance of $90 \mathrm{~mm}$ from the QCL. This combination limits the angular resolution to $1.3^{\circ}$ in the beam measurement. The beam is scanned horizontally with a step of $0.25^{\circ}$ using a stepper motor, while the scan in the vertical direction is done manually with a step of $1^{\circ}$. In Fig. 2 a two-dimensional (2D) plot of the measured beam is displayed. The radiation is concentrated within a range of roughly $\pm 20^{\circ}$ in both horizontal $(\alpha)$ and vertical $(\beta)$ angles, demonstrating a much lower divergence compared to DMW QCLs [5]. The maximum intensity appears nearly in the center $(\alpha, \beta=0)$, namely the pointing direction. Since there is no obvious sharp maximum, and there is an uncertainty of $\pm 3^{\circ}$ in the vertical direction in the setup, it is hard to determine the peak position. The maximum seems to shift slightly toward a negative $\beta$. We also observe interference fringes, superimposed on a slowly varying envelope. These can also be clearly seen in the onedimensional profiles in both horizontal and vertical directions (Fig. 3). The ringlike structure appears to be better defined in the positive vertical space ( $\beta$ $\geqslant 0$ ), namely opposite to the substrate direction.

The envelope of the far field should correspond to that of a diffraction-limited beam generated at the front facet. To analyze the data, we first compute the $2 \mathrm{D}$ mode intensity profile at the front facet and then the 2D far-field beam pattern by taking its spatial Fourier transform. The computed near- and far-field patterns are shown in Figs. 1(c) and 1(d), respectively. Despite the asymmetric near-field profile, the

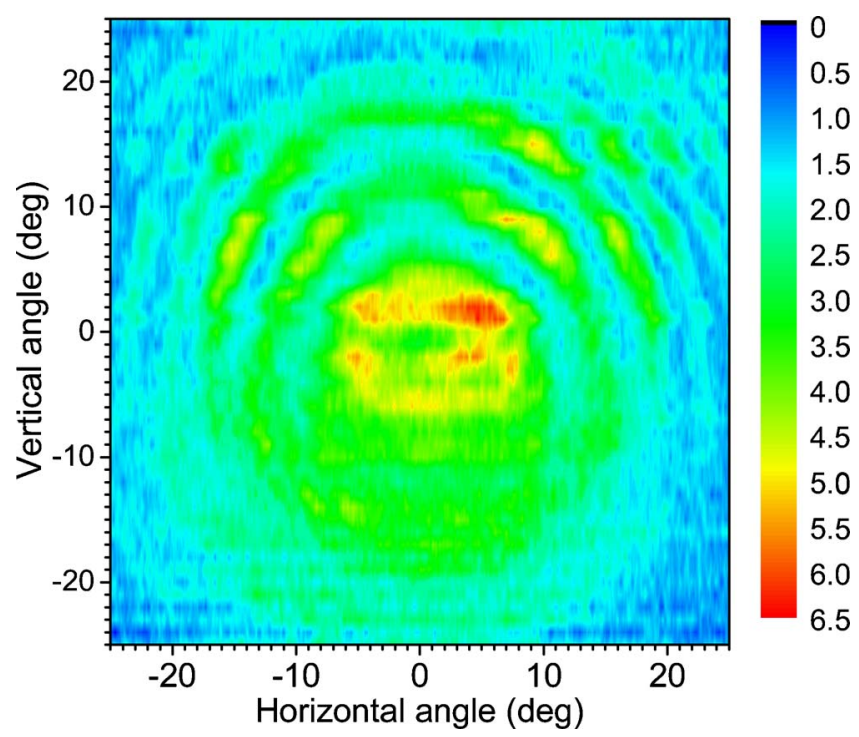

Fig. 2. (Color online) Contour plot of the measured farfield beam pattern of the $217 \mu \mathrm{m}$ wide QCL.

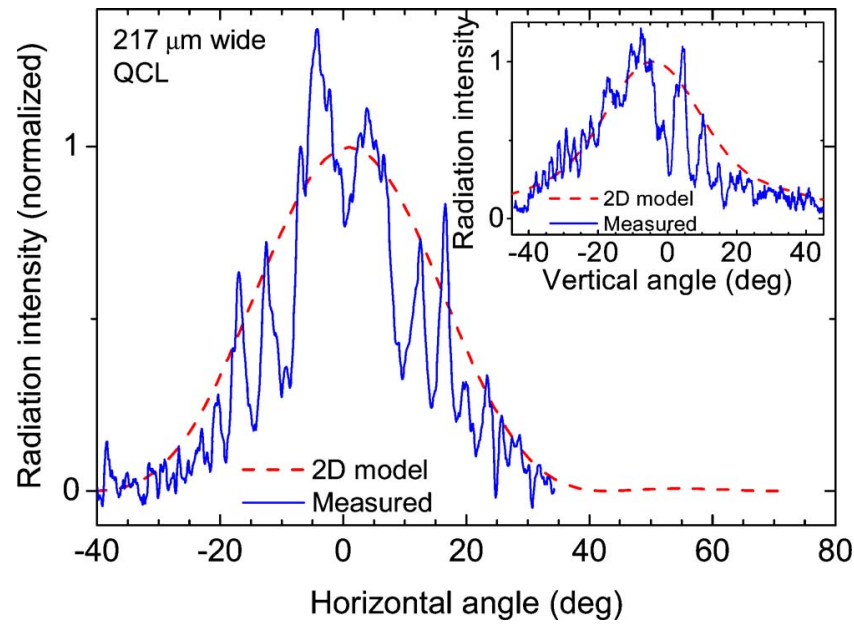

Fig. 3. (Color online) Measured beam pattern of the $217 \mu \mathrm{m}$ wide QCL in the horizontal direction, taken at zero vertical angle, together with the calculated far-field beam profile from Fig. 1(c). The inset shows the measured and calculated beam profiles in the vertical direction of the same laser. Due to a different mounting of the QCL, there could be a slight offset in the vertical direction in these measurements in comparison to the one in Fig. 2.

far-field pattern is quite symmetric with a negative offset of $4^{\circ}$ in the vertical direction. For a quantitative comparison, the calculated far-field profile in the horizontal plane is superimposed onto the measured one in Fig. 3, showing a good agreement. The calculated far-field profile in the vertical direction $(\alpha \approx 0)$ also fits the envelope of the measured one with reasonable agreement (see the inset of Fig. 3). Thus the beam divergence is determined by the near-field profile of the QCL.

Axially symmetric interference rings observed in the far field cannot however be explained by the diffraction model. Similar rings in the far field of DMW QCLs [5] were explained by the interference of radiation from longitudinally distributed sources within the laser [6]. By performing the same analysis using the model [6] we find that in the present case the angular separations between the observed fringes (nearly same in both vertical and horizontal directions) are approximately a factor of 2 smaller than what is expected from this model. A similar result is found for the $158 \mu \mathrm{m}$ wide laser, showing that the angular separations do not depend on the ridge width.

Now we turn to a heterodyne experiment using the $217 \mu \mathrm{m}$ wide QCL operated in cw mode as a $\mathrm{LO}$ and a superconducting hot electron bolometer (HEB) mixer. The mixer consists of a $2 \mu \mathrm{m}$ wide and $0.2 \mu \mathrm{m}$ long $\mathrm{NbN}$ bridge and is integrated with a tight winding spiral antenna. To determine the receiver noise temperature $\left(T_{\text {rec }}\right)$, we use a measurement setup that is similar to the one described in [2], except for a $3 \mu \mathrm{m}$ thick Mylar beam splitter and a high density polyethylene (HDPE) lens to collimate the QCL's beam. Figure 4 shows the measured $T_{\text {rec }}$ as a function of the bias voltage of the HEB mixer together with three current-voltage curves of the HEB for different absorbed LO powers [11] $(0,300$, and $380 \mathrm{nW})$. A $T_{\text {rec }}$ of $1150 \mathrm{~K}$ is obtained for $300 \mathrm{nW}$ of $\mathrm{LO}$ power and 


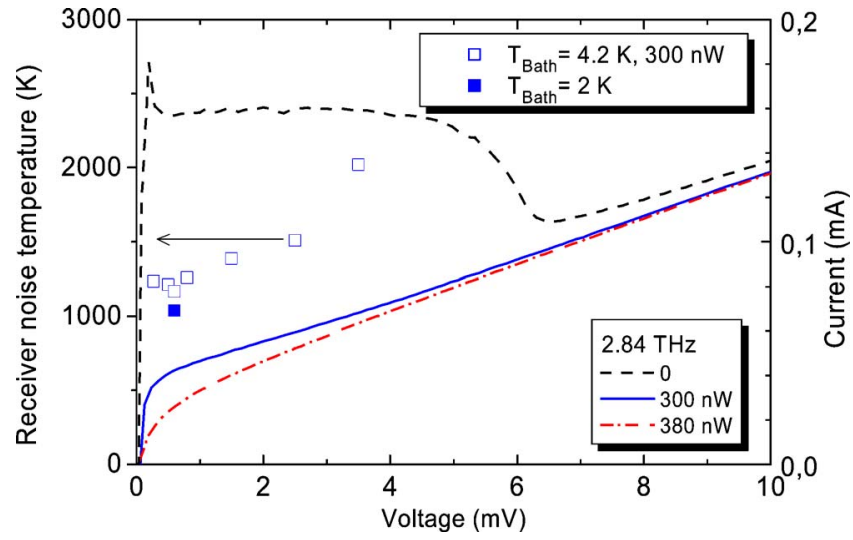

Fig. 4. (Color online) Measured receiver noise temperature (symbols, left axis) of the HEB mixer versus the bias voltage at $2.84 \mathrm{THz}$ for the optimal $\mathrm{LO}$ power $(300 \mathrm{nW})$ at $4.2 \mathrm{~K}$ and also at $2 \mathrm{~K}$. Current-voltage characteristics (curves, right axis) without and with radiation from the $217 \mu \mathrm{m}$ wide QCL.

$0.6 \mathrm{mV}$ dc bias at a bath temperature of $4.2 \mathrm{~K}$ (for the HEB). An even lower $T_{\text {rec }}$ of $1050 \mathrm{~K}$ is obtained at a reduced bath temperature of $2 \mathrm{~K}$.

We attribute the lower $T_{\text {rec }}$ partly to the slightly improved HEB mixer in the antenna and partly to the use of a thinner $(3 \mu \mathrm{m})$ beam splitter. The latter maximizes the transmission of the radiation from the hot/cold loads, which is essential to achieve ultimate receiver sensitivity. However, it reflects less power (4\%) from the LO to the HEB. The earlier measurement in DMW QCLs [2] could only use a beam splitter of $6 \mu \mathrm{m}$, which reflects more power (17\%). Allowing the use of the thinner beam splitter clearly indicates that either the beam pattern has been improved or the output power increased by using this SPW QCL in comparison to DMW QCLs [2]. When the laser is operated at maximum power, the absorbed LO power in the HEB is about $380 \mathrm{nW}$. This means that approximately $60 \mu \mathrm{W}$ of the QCL power are effectively used to pump the device. This value, calculated based on the known optical losses (22 dB), is four times higher than that found in [2]. Since the maximal output power of the QCL is only slightly higher than that in [2] $(1 \mathrm{~mW})$, we conclude that the improved beam profile is the main reason for allowing the thinner beam splitter.

In summary, we have characterized two $2.8 \mathrm{THz}$ SPW QCLs as local oscillators. We find that the QCLs have a diffraction-limited beam envelope, onto which pronounced interference fringes are superimposed. The envelope can be described by the far-field pattern computed in the diffraction limit from the mode profile at the front facet of the laser. Such a beam allows coupling of the radiation to the HEB mixer by employing a very thin beam splitter and simplified optics. We obtained a receiver noise temperature of $1050 \mathrm{~K}$.

We acknowledge S. Paprotskiy and X. Gu for helping with beam measurements, as well as A. Andronico, E. E. Orlova, T. O. Klaassen, A. Baryshev, J. J. A. Baselmans, and Z. Q. Yang for useful discussions. We also thank H. Hoevers for his support, and the EU through RadioNet, Teranova, and the PASR 2004 Project Terasec for financial support.

\section{References}

1. R. Köhler, A. Tredicucci, F. Beltram, H. E. Beere, E. H. Linfield, A. G. Davies, D. A. Ritchie, R. C. Iotti, and F. Rossi, Nature 417, 156 (2002).

2. J. R. Gao, J. N. Hovenier, Z. Q. Yang, J. J. A. Baselmans, A. Baryshev, M. Hajenius, T. M. Klapwijk, A. J. L. Adam, T. O. Klaassen, B. S. Williams, S. Kumar, Q. Hu, and J. L. Reno, Appl. Phys. Lett. 86, 244104 (2005).

3. H.-W. Hübers, S. G. Pavlov, A. D. Semenov, R. Köhler, L. Mahler, A. Tredicucci, H. E. Beere, D. A. Ritchie, and E. H. Linfield, Opt. Express 13, 5890 (2005).

4. B. S. Williams, S. Kumar, H. Callebaut, Q. Hu, and J. L. Reno, Appl. Phys. Lett. 83, 2124 (2003).

5. A. J. L. Adam, I. Kašalynas, J. N. Hovenier, T. O. Klaassen, J. R. Gao, E. E. Orlova, B. S. Williams, S. Kumar, Q. Hu, and J. L. Reno, Appl. Phys. Lett. 88, 151105 (2006).

6. E. E. Orlova, J. N. Hovenier, T. O. Klaassen, I. Kašalynas, A. J. L. Adam, J. R. Gao, T. M. Klapwijk, B. S. Williams, S. Kumar, Q. Hu, and J. L. Reno, Phys. Rev. Lett. 96, 173904 (2006).

7. S. Kohen, B. S. Williams, and Q. Hu, J. Appl. Phys. 97, 053106 (2005).

8. E. Bründermann, M. Havenith, G. Scalari, M. Giovannini, J. Faist, J. Kunsch, L. Mechold, and M. Abraham, Opt. Express 14, 1829 (2006).

9. S. Barbieri, J. Alton, H. E. Beere, J. Fowler, E. H. Linfield, and D. A. Ritchie, Appl. Phys. Lett. 85, 1674 (2004).

10. S. Barbieri, J. Alton, C. Baker, T. Lo, H. Beere, and D. Ritchie, Opt. Express 13, 6497 (2005).

11. H. Ekström, B. S. Karasik, E. L. Kollberg, and K. S. Yngvesson, IEEE Trans. Microwave Theory Tech. 43, 938 (1995). 